\title{
Approximation of Continuous Media Models for Granular Systems Using Cellular Automata
}

\author{
M. Pla-Castells, I. García, and R. J. Martínez \\ Laboratorio de Simulación y Modelado \\ Instituto de Robótica, Universidad de Valencia \\ P.O. Box 2085, 46071 Valencia Spain, \\ Marta.Pla@uv.es
}

\begin{abstract}
In this paper a new cellular automata model suitable for granular systems simulation is presented. The proposed model is shown to be equivalent to a particularization of the well known BCRE model of granular systems and a correspondence between the parameters of the presented model and the BCRE model is also set, allowing to fit these parameters for a given system. The model has the advantage over other cellular automata models of being more realistic in the behavior of the surface of heaps and slopes. The dynamics of the CA is analyzed in order to confirm that it also has one of the most important features of these systems, $1 / f$ noise.
\end{abstract}

\section{Introduction}

Granular systems behavior has been widely studied during the last decades, due to its many applications to industry. They show a complex behavior that cannot be easily classified in one of the usual matter states; solid, gas or fluid.

The traditional approach for studying its dynamics has been based in fluids models, describing the flow of granular systems and the formation of heaps. However granular systems show characteristics, such as the appearance of macroscopic patterns or avalanches, that cannot be properly described using this approach. By the other side, Cellular Automata (CA) have also been used for granular systems modelling. Several works have been published that use CA models to study the statistical properties of granular systems [1-3]. However the formation of heaps and the way the size of a pile of matter evolves is better described by fluid models.

When simulating the behavior of a granular system in a computer graphics application, the most relevant part is the visualization of the evolution of the external surface of the pile along time [4]. Models based on fluid dynamics do not suit quite well to such applications, since their computational cost makes them difficult to apply to these kind of real-time simulations. In opposite, CA based models describe the granular system as a grid, therefore, as has been shown in [5], they can be solved very efficiently.

In previous works $[5,6]$ some of the CA models found in the bibliography $[1$, 7] were reviewed in order to obtain a more realistic heap and slope formation. 
The main problem of these models is their anisotropy, which is specially notable when a heap is formed by the addition of matter in a single point [6]. The resulting heap has polygonal section, instead of being conic.

Thus, on the one hand continuous models are not suitable for real-time simulation. On the other hand, CA-based models fit well the requirements of this kind of application, but do not show a behavior realistic enough. The objective of this work will be to obtain a CA-based model with a behavior somehow equivalent to that of continuous models. This model fills the need of a realistic and computationally inexpensive model.

In order to achieve this, a model that is shown to be locally equivalent to an existent continuous model is proposed. The model presented by Bouchaud, Cates, Prakash and Edwards [8] (also known as BCRE model) will be used as the starting point, because of its simplicity and its intuitive description.

The BCRE Model A granular system can be considered as a system with two layers; the standing layer, that forms the slope or the heap of the system, and the rolling layer, that is a thin layer that flows on the surface of the slope [9]. The BCRE model [8] uses this schema and provides a set of equations to describe the evolution of these layers' thickness. The model can be formulated in one dimension as follows: the system has two state variables, the height of the static layer, $s(x, t)$ and the height of the rolling layer, $r(x, t)$. The variation of these variables along time is expressed by the set of equations

$$
\begin{aligned}
& r_{t}=v r_{x}-\gamma\left(\alpha-\left|s_{x}\right|\right) r \\
& s_{t}=\gamma\left(\alpha-\left|s_{x}\right|\right) r
\end{aligned}
$$

where $\alpha$ is the so called angle of repose of the system, $\gamma$ is a parameter that expresses the rate of matter transfer between layers and $v$ is the speed of the rolling layer that is considered constant.

Equations (1) express that the thickness of both layers will depend on two major processes: on the one hand the transfer of matter from the rolling layer to the resting layer or vice-versa, and on the other hand the exchange of matter at each point of the rolling layer with its surroundings.

This model was extended to 2 dimensions [10] leading to a set of partial differential equations on the variables $r(x, y, t)$ and $s(x, y, t)$

$$
\begin{aligned}
& r_{t}=v \nabla(r \nabla s)-\gamma(\alpha-|\nabla s|) r \\
& s_{t}=\gamma(\alpha-|\nabla s|) r
\end{aligned}
$$

using the same notation as in equations (1). Equations (2) define the 2-dimensional continuous model that will be used later in this paper.

Following, the update rule that defines the new model will be presented, and the relation between this new model and the BCRE will be explained. In the third section, some properties of the new model will be shown in order to demonstrate that, despite the goal of this work, the main statistical property of most CA models is kept. 


\section{Description of the Model}

In order to define an update rule for a CA model that reflects the behavior of a continuous surface, some simplifications to the two layer description of the granular system that was seen before have to be done.

To define the new model we will work under two main assumptions. The first one is that the thickness of the rolling layer is constant, being $h=r+s$ the height of the system at every point. The second assumption is that the surface of the granular system $h(x, y)$ is a two times differentiable function.

A CA on an $N \times N$ square grid is considered. This grid represents the plane on which the granular system is laying. The value of each cell $h(i, j) \in \mathbb{R}$ will represent the height of the system on the cell's center $\left(x_{i}, y_{j}\right)$. The set of points $\left\{\left(x_{i}, y_{j}, h(i, j)\right)\right\}_{i j}$ is a discretization of the surface $\{(x, y, h(x, y))\}$. Notice that, although the update rule is defined using the derivatives of $h(x, y)$, an expression in terms of the CA variables can be easily obtained.

The update rule is as follows. For each cell, $(i, j)$, the gradient $\nabla h\left(x_{i}, y_{j}\right)$ is computed. If the angle of the slope $\arctan (|\nabla h|)$ is lower than the repose angle threshold $\alpha$, the value of cell $(i, j)$ remains unchanged. On the other case, if $\arctan (|\nabla h|)>\alpha$, the height $h(i, j)$ is reduced $z_{+} \cdot\left(h_{x}+h_{y}\right), h(i+1, j)$ is increased $z_{+} \cdot h_{x}$ and $h(i, j+1)$ is increased $z_{+} \cdot h_{y}$, that is:

$$
\begin{aligned}
h(i, j) & \leftarrow h(i, j)-z_{+} \cdot\left(h_{x}(i, j)+h_{y}(i, j)\right) \\
h(i+1, j) & \leftarrow h(i+1, j)+z_{+} \cdot h_{x}(i, j) \\
h(i, j+1) & \leftarrow h(i, j+1)+z_{+} \cdot h_{y}(i, j)
\end{aligned}
$$

where $z_{+}$is a parameter of the model which indicates the velocity of flowing matter, and $h_{x}, h_{y}$ are the partial derivatives of $h(x, y)$.

From the assumption that the values of the automata are a discretization of the surface of the system, the value of $\nabla h$ can be obtained taking the approximation of the derivatives by differences

$$
\begin{aligned}
& h_{x}(i, j)=\frac{\partial h}{\partial x}\left(x_{i}, y_{j}\right)=\frac{h\left(x_{i+1}, y_{j}\right)-h\left(x_{i}, y_{j}\right)}{x_{i+1}-x_{i}} \\
& h_{y}(i, j)=\frac{\partial h}{\partial y}\left(x_{i}, y_{j}\right)=\frac{h\left(x_{i}, y_{j+1}\right)-h\left(x_{i}, y_{j}\right)}{y_{j+1}-y_{j}} .
\end{aligned}
$$

This rule is based in the fact that the gradient vector field $-\nabla h(x, y)$ indicates the direction of maximum slope at every point in the surface of the heap. When a cell is updated, if the angle of the slope is higher than the repose angle, the matter flows following the direction of the strongest slope.

\subsection{Relation with the BCRE}

Once the update rule has been set, the relationship between the behavior of this CA model and the BCRE continuous model will be shown. In order to obtain 
this relationship the variation of both models at a given point $\left(x_{i}, y_{j}\right)$, and along a fixed time interval $\delta t$, will be obtained. It will be shown that, under certain assumptions, the difference between the models tends to zero if the time interval and the cell size are reduced.

The system evolution after time $\delta t$ is represented in the CA by an update of the grid using the specified rule. The variation of the height of a cell $(i, j)$ will depend on the update of the cell $(i, j)$ itself, and on the update of cells $(i-1, j)$ and $(i, j-1)$, as they can drop grains into cell $(i, j)$. In a situation in which these three updates are not null, the difference between the height of a cell of the automata, before and after the update, is

$$
\Delta h_{C}=z_{+}\left(h_{x}(i, j)+h_{y}(i, j)-h_{x}(i-1, j)-h_{y}(i, j-1)\right)
$$

To see the variation of the BCRE model after time $\delta t$, the thickness of the rolling layer $r$ will be considered constant in an open set $A$ including a given point $\left(x_{i}, y_{j}\right)$. According to equation $(2)$, in $\left(x_{i}, y_{j}\right)$ the variation of the total height of the system, $h=r+s$, is

$$
h_{t}=r_{t}+s_{t}=v \nabla(r \nabla s)
$$

that, as $r$ is constant around $\left(x_{i}, y_{j}\right)$, equals

$$
=\operatorname{vr} \nabla(\nabla s)=\operatorname{vr} \nabla(\nabla h)=\operatorname{vr}\left(h_{x x}+h_{y y}\right) .
$$

Developing the Taylor series of $h\left(x_{i}, y_{j}, t\right)$ as a function of time, one obtains

$$
\begin{aligned}
\Delta h_{B}=h(t+\delta t)-h(t) & =h_{t} \delta t+R(\delta t)^{2} \\
& =\delta t \operatorname{vr}\left(h_{x x}+h_{y y}\right)+R(\delta t)^{2} .
\end{aligned}
$$

In a similar way, Taylor series of $h_{x}\left(\cdot, y_{j}, t\right)$ considered as function of $x$ and $h_{y}\left(x_{i}, \cdot, t\right)$ considered as function of $y$ give

$$
\begin{aligned}
& h_{x x}\left(x_{i}, y_{j}\right)=\frac{h_{x}\left(x_{i+1}, y_{j}\right)-h_{x}\left(x_{i}, y_{j}\right)}{x_{i+1}-x_{i}}+R_{1}\left(x_{i+1}-x_{i}\right) \\
& h_{y y}\left(x_{i}, y_{j}\right)=\frac{h_{y}\left(x_{i}, y_{j+1}\right)-h_{y}\left(x_{i}, y_{j}\right)}{y_{j+1}-y_{j}}+R_{2}\left(y_{j+1}-y_{j}\right) .
\end{aligned}
$$

From Equation (9), assuming $x_{i}-x_{i-1}=y_{j}-y_{j-1}=d$

$$
\begin{aligned}
h(t+\delta t)-h(t)= & \delta t \operatorname{vr}\left(\frac{h_{x}(x+d, y)-h_{x}(x, y)}{d}\right. \\
& \left.+\frac{h_{y}(x, y+d)-h_{y}(x, y)}{d}+\left(R_{1}+R_{2}\right) d\right) \\
& +R(\delta t)^{2} .
\end{aligned}
$$

Expressions (6) and (12) give respectively the variation of the proposed CA model, $\Delta h_{C}$, and of the BCRE model, $\Delta h_{B}$, after an interval of time $\delta t$. Taking

$$
z_{+}=\frac{\delta t v r}{d}
$$


and subtracting equalities 6) and (12) one gets

$$
\Delta h_{C}-\Delta h_{B}=\delta t \operatorname{vr}\left(R_{1}+R_{2}\right) d+R(\delta t)^{2}
$$

that represents the difference of the evolution of both models at point $\left(x_{i}, y_{j}\right)$.

Therefore, considering the situation in which the thickness of the rolling layer in the BCRE model is constant around $\left(x_{i}, y_{j}\right)$, and in which the three cells $(i, j)$, $(i-1, j)$ and $(i, j-1)$ are effectively updated (i.e. cell $(i, j)$ is in a flowing region), both models converge locally if $\delta t$ and $d$ tend to zero.

Notice that the assumption of a constant rolling layer implies that the matter flow is not null in a certain region around point $\left(x_{i}, y_{j}\right)$. In the terms of the CA representation this means that, for $d$ small enough, the update of the four neighbors of cell $(i, j)$ is not null. This is, precisely, which is the assumption made over the CA model to get equation (14).

Thus, as a conclusion, equation (14) expresses that in a point around which the variation of the rolling layer is neglectable, both models lead to the same behavior.

\section{Avalanches and $1 / f$ Noise}

Granular systems are considered a paradigmatic example of systems that evolve to a state called Self Organized Criticality (SOC) [11]. When in this regime, the system remains stable but near to unstable states. When it is perturbated, the system evolves through a set of unstable states until a new stable state is reached. This behavior is usually illustrated by a slope of sand into which grains are dropped randomly. When the slope is higher than a certain angle, the grains start rolling and an avalanche takes place until the system gets relaxed.

Some numerical experiments have been prformed in order to test if one of the most characteristic marks of this behavior, the so called $1 / f$ noise, is also present in the model. $1 / f$ noise refers to the frequency spectrum of certain events such as avalanches: in a critical system the frequency at which a certain event happens is proportional to the inverse of its magnitude.

Reproducing the tests performed by several authors [1, 12-14], the frequency of the size of avalanches along a slope, and the correlation between the frequency and the size have been obtained. As a measure of size three values have been chosen; its length as the number of steps that lasts until the system stops again, the area of the avalanche or the number of cells it affects, and the mass displaced, that is the sum of all the matter transfer at every update. Figure 1 shows the power spectra of the measured events.

They have been approximated by a theorical $1 / f^{-\beta}$ function and the correlation of the data have been calculated. The results of this tests are: for the length of the avalanches, $\beta=-6.255718$ with a correlation $r=0.862181$, for the area, $\beta=-3.375581$ and $r=0.933205$ and for the displaced mass, $\beta=-7.518815$ and $r=0.960261$.

The correlation of the measurements of frequency to the theoretical function indicates that the well known behavior of granular systems as critical systems 

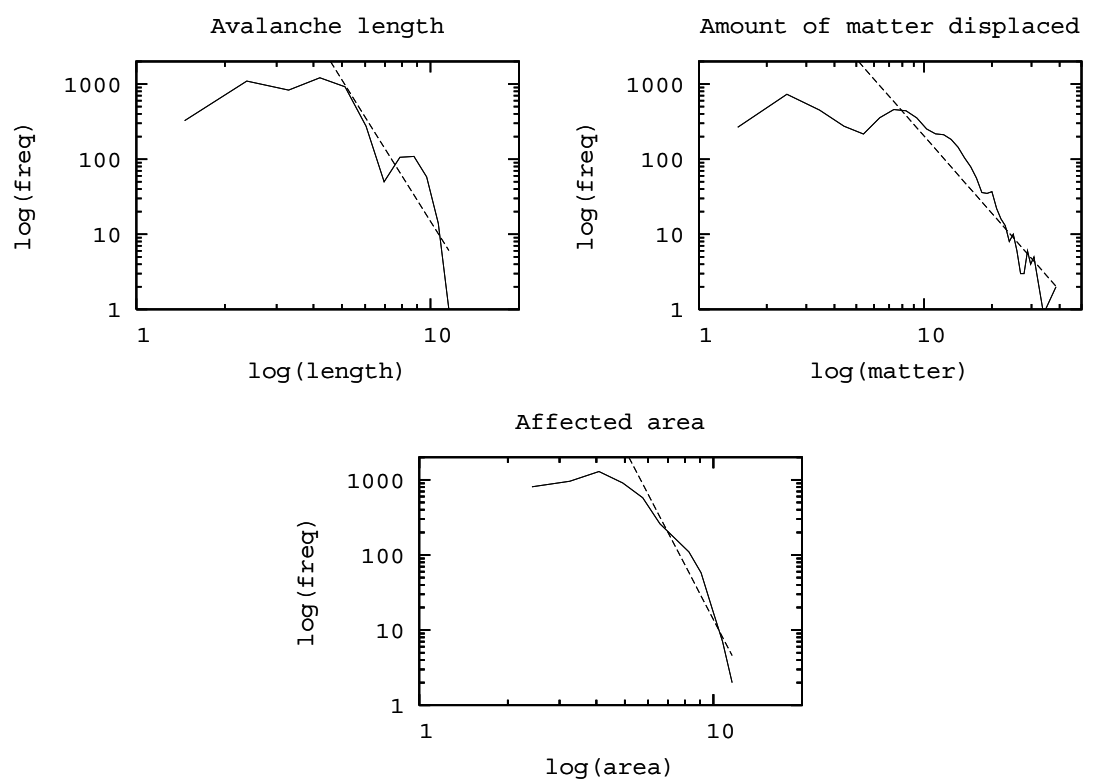

Fig. 1. Frequency spectra for the length, the area and the amount of matter affected by an avalanche, showing the existence of an $i / f^{-\beta}$ relationship. The dashed line shows the regression of the data to a function of that family. 
is possible in the presented model. This fact, together with the results obtained in section 2.1 make the model a good tool for the analysis and representation of granular systems.

\section{Application of the Model}

The integration of the developed model in a more complex application has been done during the development of the Mobile Crane Simulator UVSim, that is currently used in Valencia Harbor. The model presented in this paper is used as a dynamic model for the behavior of bulk material within the complete dynamic model of the virtual environment in the simulator.

In addition to the $3 \mathrm{D}$ graphics representation of the system, some interaction modes have been defined in order to allow the manipulation of the bulk material. Further details on the implementation and on the 3D representation of the system using the CA model can be found in [5].

\section{Conclusions}

In this paper a model addressed to real-time simulation of granular systems that encloses properties of both continuous media models and CA models has been defined. On the one hand, it has been shown that the evolution of the surface of the system is equivalent to the well known BCRE model. On the other hand, some experiments have been done in order to see that the model also keeps at least one of the most characteristic statistical properties of CA.

The evolution of the model has been analyzed at an arbitrary point; it has been shown that the local behavior of the surface is the same that in the BCRE model under the assumption that the rolling layer of the system does not vary substantially near the analyzed point. It has been also stated a relationship between the parameters of both models. The amount of matter displaced $z_{+}$ is in equation (13) and the threshold of the automata is set as $\arctan \alpha$. This correspondence allows a direct parameterization of the new model taking as a starting point the parameters of the BCRE model.

Hence, the model fulfills the main requirements stated in Section 1; it behaves as one of the most extended continuous models for granular systems, and has the advantage that the $\mathrm{CA}$ implementation is suitable for real-time applications.

Also it has been shown that, although the model behaves locally as a continuous media model, the statistical behavior of the model is consistent with previous CA models. The power spectra of three variables related to avalanches have been obtained, showing that $1 / f$ noise, a paradigmatic characteristic of granular systems, can be present in the dynamics of the model.

It can be concluded that the model that has been presented in this work covers the main goals proposed in this paper, providing an improvement of previous CA models.

As future research, the model should be completed to fit the BCRE model in the general case, and perhaps other continuous models should also be translated 
to a CA model. The analysis of the statistical properties of the model has to be completed in order to determine the different regimes of its dynamics. The possibility of pattern formation can also be studied simulating the experiments usually performed with real granular systems. These works will provide a more robust evidence of the validity of the CA model for granular systems simulation.

\section{References}

1. Chen, C. C., den Nijs, M.: Directed avalanche processes with underlaying interface dynamics. Physical Review E. 66 (2002).

2. Prado, C., Olami, Z.: Inertia and break of self-organized criticality in sandpile cellular-automata models. Phys. Rev. A 45 (1992) 6665-6669

3. Nerone, N., Gabbanelli, S.: Surface fluctuations and the inertia effect in sandpiles. Granular Matter. 3 (2001) 117-120

4. Müller, M., Charypar, D., Gross, M.: Procedural modeling and animation: Particlebased fluid simulation for interactive applications. ACM SIGGRAPH/Eurographics Symposium on Computer Animation (2003) 154-159

5. Pla-Castells, M.: Nuevos modelos de sistemas granulares basados en autómatas celulares para simulación en tiempo real. MSc Thesis. Escuela de Ingenierías, Universidad de Valencia. (2003)

6. Pla-Castells, M., García, I., Martínez, R.J.: Visual Representation Of Enhanced Sand Pile Models. Industrial Simulation Conference. Universidad Politécnica de Valencia, Valencia, Spain (2003) 141-146

7. Christensen, K., Olami, Z., Bak, P.: Deterministic $1 / f$ noise in nonconservative models of self-organized criticality. Phys. Rev. Letters 68 (1992) 2417-2420

8. Bouchaud,J. P.,Cates,M. E ,Prakash,J. R., Edwards, S. F.: A Model for the Dynamics of Sandpile Surfaces. J. Phys. I France. 4 (1994) 1383-1410

9. Aradian, A., Raphael, E., de Gennes, P. G.: Surface flows of granular materials: a short introduction to some recent models. Comptes Rendus Physique, 3 (2002) 187-196

10. Hadeler, K. P., Kuttler, C.: Dynamical models for granular matter. Granular Matter.2 (1999) 9-18

11. Bak, P., Tang, C., Wiesenfeld, K.: Self-organized criticality Phys. Rev. A, 38 (1988) 364-374

12. Kadanoff, L. P., Nagel, S. R., Wu, L., Zhou, S.: Scaling and universality in avalanches. Phys. Rev. A. 39 (1989) 6524-6537

13. Dorso, C. O. , Dadamia, D.: Avalanche prediction in Abelian sandpile model. Physica A: Statistical Mechanics and its Applications. 308 (2002) 179-191.

14. Kertesz, J., Torok, J., Krishnamurthy, S., Roux, S.: Slow dynamics in selforganizing systems. Physica A: Statistical Mechanics and its Applications. 314 (2002) 567-574. 